\title{
GLAD!
}

Revue sur le langage, le genre, les sexualités

$06 \mid 2019$

Varia

\section{Discours féministe sur le génocide rwandais dans deux romans africains d'Afrique noire francophone : entre devoir de mémoire et écriture- femme}

Feminist Discourse about the Rwandan Genocide in two Francophone Novels from Sub-Saharan Africa: between Duty of Memory and Woman-Writing

\section{Didier Brou Anoh}

\section{OpenEdition}

\section{Journals}

Édition électronique

URL : http://journals.openedition.org/glad/1524

DOI : $10.4000 /$ glad. 1524

ISSN : 2551-0819

\section{Éditeur}

Association GSL

\section{Référence électronique}

Didier Brou Anoh, «Discours féministe sur le génocide rwandais dans deux romans africains d'Afrique noire francophone : entre devoir de mémoire et écriture-femme », GLAD! [En ligne], 06 | 2019, mis en ligne le 01 juillet 2019, consulté le 17 décembre 2020. URL : http://journals.openedition.org/glad/1524 ; DOI : https://doi.org/10.4000/glad.1524

Ce document a été généré automatiquement le 17 décembre 2020.

\section{cc) (†)}

La revue GLAD! est mise à disposition selon les termes de la Licence Creative Commons Attribution -

Pas d'Utilisation Commerciale - Pas de Modification 4.0 International. 


\section{Discours féministe sur le génocide rwandais dans deux romans africains d'Afrique noire francophone : entre devoir de mémoire et écriture-femme}

Feminist Discourse about the Rwandan Genocide in two Francophone Novels from Sub-Saharan Africa: between Duty of Memory and Woman-Writing

Didier Brou Anoh

\section{Introduction}

1 Bien que sorti du complexe dans lequel on l'avait enfermé, le féminisme fait encore débat sur son origine que certains attribuent au philosophe Ch. Fourier ou encore à A. Dumas. Le mot trouve sa définition dans le besoin d'émancipation qui anime les femmes (occidentales) et le souci de mettre fin à leur subordination légale aux hommes, rappelant la célèbre formule beauvoirienne : «On ne naît pas femme, on le devient » (Beauvoir 1976[1946] : 13).

2 Le féminisme s'est affirmé en Occident déjà dans le dernier tiers du vingtième siècle, avec des auteures défendant la liberté des femmes, comme $\mathrm{M}$. Wittig (pionnière du Mouvement de Libération des Femmes), qui a écrit Le corps lesbien (1972), et s'est étendu, ces dernières années au reste du monde. On note une pluralité de manifestations du féminisme qui vont des individualités à des regroupements multiformes, des plus pacifistes au plus radicaux, notamment "chiennes de Garde », "Ni pute ni soumise», "Osez le féminisme», "SOS sexisme», «Femen", «Tumultueuses », «\#SiOnMetue », etc. 
3 De plus en plus, les débats féministes tournent non plus autour du féminisme qui met en question la structure duelle et inégalitaire qui régit les rapports de sexes, mais des féminismes dans lesquels émergent diverses sensibilités : féminisme Black, féminisme judaïque, chrétien ou musulman, intersectionnel, postmoderne, postcolonial, etc. Transposé dans la littérature, le débat, vieux de plusieurs années, continue de se cristalliser autour de l'existence ou non d'une écriture féminine féministe qui consacre les « mots aux maux de la femme» (Dah 2018 : 335-355).

4 En s'appropriant ce terme dans ses formes diverses pour traduire leurs sentiments, des auteures africaines mettent en jeu la problématique du Sujet féminin et sa centralité dans le jeu narratif. La question de l'intimité et de l'extimité du féminin que S. Tisseron désigne comme étant le "processus par lequel des fragments du soi intime sont proposés au regard d'autrui » (2011:84), du corps (de la femme) auquel des « analystes féminins » ont donné « ses lettres de noblesse » (Probyn 1992 : 35), pose les fondements d'une écriture dont le postulat de base est la condition des femmes dont la représentation imprime des identifications afférentes (féminisme-s, féminité, identité féminine...)

5 V. Tadjo et M. Ilboudo sont au nombre de ces femmes africaines qu'O. Caseneuve considère comme des Femmes rebelles (1996), parce que leur écriture parcourt les lieux communs des réalités perçues comme ineffables. Ayant participé au projet Fest'Africa qui a regroupé plusieurs auteurs africains désireux de comprendre le massacre de masse des Tutsi par les Hutu en 1994 au Rwanda, sous le régime du Hutu Power, elles ont contribué à marquer la mémoire du génocide.

6 Leurs écrits sont un combat contre une expérience et une politique de déshumanisation, un projet d'effacement (c'est la définition qu'on pourrait donner au génocide rwandais) de la minorité tutsi du Rwanda. Ce génocide, survenu après que l'avion du président Juvénal Habyarimana (dictateur hutu au pouvoir depuis 1990) a été abattu par les maquisards du Front patriotique rwandais (essentiellement tutsi), pourrait, en effet, être défini comme tel.

7 Le langage utilisé pour exprimer l'horreur du génocide correspond à un véritable cri de cœur de femmes meurtries par la cruauté des hommes mais aussi des femmes accusées de crime de viol, à l'instar de Pauline Nyiramasuhuko, ministre de la famille et de la promotion des femmes pendant le génocide, qui a supervisé et encouragé le massacre des Tutsi, avec cette injonction qu'elle ne cessait de répéter aux Interahamwe (ceux qui tuent ensemble) : " Avant de tuer les femmes, vous devez les violer », et reprise comme titre de son ouvrage par S. Ricci (2014).

8 Au cœur de leurs matières narratives qui essaiment une écriture du dégout, un discours de la révolte, on retrouve les faibles, les enfants, les vieillards, les femmes. Leur présence dans le récit entraine une tension du récit pour traduire les maux de «notre humanité en danger» (Tadjo 2000: 135). "Féministes [africaines] engagées" (Gallimore 1997 : 50), à l'image de C. Beyala, F. Diome, témoins indirects du massacre des Tutsi (leurs textes sont basés sur des récits de témoignage écrits et filmiques, des comptes rendus, des archives, etc.), elles font une prise de parole en tant que Sujets trans-individuels pour transcrire le génocide perpétré envers les Tutsi du Rwanda.

9 Leur témoignage du génocide traduit un glissement de la parole vers un discours de la révolte et de la véhémence. Une telle posture entraine un certain nombre de questions liées à ce qui particularise le récit du génocide par des femmes, aux spécificités de sa 
mise sur la scène de la littérature : comment, par l'écriture, deux femmes africaines traduisent l'extrême violence comme celle du génocide rwandais? Quelle est la spécificité du langage pour décrire le génocide perpétré envers les Tutsi du Rwanda ? À quoi répond une telle stratégie, et quelle est sa répercussion sur le texte narratif ?

Il n'y pas meilleure méthode pour aborder une telle étude que l'analyse du discours émotionnel qu'A. Gefen et $\mathrm{E}$. Bouju considèrent comme étant la recherche du « dédain [...] pour des problématiques apparaissant comme insuffisamment formalistes et trop "psychologisantes" " (2013: 5), tant il sera question d'inscrire le témoignage du génocide rwandais dans la perspective du récit de la véhémence, de la violence et des tourments qui agitent les sociétés africaines modernes.

11 Notre hypothèse est que certaines auteures africaines, profondément affectées par la violence qui touche les sociétés africaines (les femmes en particulier), extériorisent leur colère dans les lignes du roman, au moyen d'un langage violent, cru. Leurs textes narratifs renvoient l'image de femmes africaines militantes, dont l'écriture, comme le perçoit B. Rigolt expliquant Paroles de femmes d'A. Leclerc (1974), est un « renouvellement des savoirs, qui passe par l'affirmation du féminin [...] » (2016). Cette étude s'attachera à montrer essentiellement les traits et les enjeux du langage féministe postcolonial marqué par une aptitude à lire le monde, " à lire les représentations des femmes en littérature en prêtant attention à la fois au sujet et au moyen de la représentation » (Barhi $2010: 27-54)$, lesquelles s'appuient sur le point de vue postcolonial dans ses grandes lignes (différence de pouvoir sur le statut social, politique, culturel...), dans le contexte du génocide rwandais.

\section{Deux femmes, une résidence d'écriture : pour quoi faire?}

Qu'est ce qui a bien pu pousser deux femmes à effectuer un voyage au Rwanda après le génocide de 1994, quand on sait avec M. Fusaschi qu'« être femme sur le terrain peut avoir des conséquences multiples" (2013:29-40). Grâce à la résidence d'écriture (une opportunité) obtenue dans le cadre du projet Fest'Africa, V. Tadjo et M. Ilboudo voulaient " disséquer les mécanismes de la haine », comprendre " les actes qui scellent les trahisons », « les gestes qui enclenchent la terreur » (Tadjo 2000 : 135).

Elles avaient surtout l'intention de comprendre (pour donner un nom à ce qui venait de se produire) pourquoi le mari (hutu) a tué sa femme (tutsi), pourquoi le prêtre (hutu) a livré ses fidèles (tutsi) venus se réfugier dans sa chapelle, aux miliciens interahamwé, etc. En somme, elles étaient soucieuses de répondre à la question suivante que pose la quatrième de couverture de L'ombre d'Imana : "Comment vit-on là où sa femme, son bébé, son père ont péri, qu'il fût tué par un voisin ou un inconnu ?» (op cit : 142).

Leur récit du génocide se particularise par le choc des mots et la révolte face à la brutalité de l'homme. Face au discours de la haine porté par des miliciens entrainés pour tuer, elles proposent « un contre-discours» (Gallimore 2001: 79-98) qui rompt avec le silence et porte selon A. Bassolé Ouédraogo, « les marques de l'ostracisme et se confronte au discours hégémonique patriarcal » $(1998: 2)$.

Dans la peau de femmes sous le choc, elles tentent de redonner des visages, des voix et de la vie à tous ceux qu'elles rencontrent durant leur parcours du Rwanda pour comprendre l'une des tragédies africaines les plus cruelles : le génocide perpétré envers 
les Tutsi du Rwanda. En se mettant (quelquefois) elles-mêmes en scène dans le récit à travers une auto-réflexion, et en donnant la parole à ceux qu'elles rencontrent durant la tentative de compréhension du massacre de masse des Tutsi du Rwanda, les deux auteures instaurent un regard personnel sur le génocide, et font une lecture de l'autre dans la posture à la fois de bourreau et de victime.

Au-delà du récit présenté sous les traits d'une fiction-témoignage, V. Tadjo et $\mathrm{M}$. Ilboudo posent un regard lourd sur le génocide rwandais qui revêt, selon M. Faulkner, «un triple sens»: celui du "voyage physique à travers le pays des mille collines, voyage introspectif d'un individu qui confronte une histoire violente et le voyage dans l'écriture, en l'occurrence l'exploration des limites de l'emploi du langage et des formes littéraires pour représenter le génocide » (2012: 253-255).

tens du voyage dont parle $\mathrm{M}$. Faulkner repose sur une double posture de l'écriture génocidaire: le témoignage et l'engagement. Témoignage parce que le récit du génocide porte la voix des victimes tout comme celle des deux auteures. Mieux, renchérit V. Bonnet, en témoignant, «l'écriture devient acte de résistance face à la violence qui broie progressivement les repères sociaux temporels ", là où " les mots [en ce qui les concerne] construisent un refuge » (2008: 105-113). Engagement dès lors que l'écriture (engagée), sous l'angle de perception de Sartre, « rappelle l'importance de réaliser l'unité existentielle du moi, à la fois citoyen et écrivain, en abordant des questions sociales dans les textes littéraires » (Karegeye 2009 : 21-32).

Cette écriture tente de concilier les exigences esthétiques avec les horizons d'attente de la société, en présentant le génocide comme un tourment de l'Afrique. Entre la réalité dicible de la violence et le jeu de la fiction, on retrouve l'écrivain (la femme notamment) dans son rôle de critique et d'éveilleur de conscience. Selon J.P. de Sartre, sa parole devient "action " (Sartre $2002: 28$ ) contre un monde de tension et de crise, surtout que «les textes "'rwandais"” sur le génocide des Tutsi écrits par des femmes rescapées forment les témoignages les plus connus et surtout les plus significatifs » (Karegeye 2014 : 328-349).

Dans leur approche du génocide, le Rwanda est une terre à « exorciser » au moyen de l'écriture, qui est à l'écoute des situations de crises extrêmes à partir desquelles la parole du témoin devient un objet de réflexion sur le sens de la vie. Sans tomber dans le piège du procès post-génocide dans lequel s'installe quelquefois une confusion entre le rôle du bourreau et celui de la victime, l'écriture de V. Tadjo et de M. Ilboudo tente d'interroger le génocide et ses zones d'ombre, pour créer un imaginaire crédible chez le lecteur, avec en toile de fond, cette interrogation de S. Mukayiranga (2003) : «Sommesnous entrés dans une ère de négation?»

Selon J. Semujanga, la parole qui guide l'écriture de V. Tadjo « a un but didactique. Elle veut apprendre quelque chose au lecteur au sujet du génocide en en faisant un thème de réflexion sur la contamination du monde par la haine de l'autre» (2008:156). Celle de M. Ilboudo, tout en inscrivant le génocide rwandais dans le lot des grandes tragédies de l'Histoire humaine, «se construit autour d'une interrogation centrale: est-il possible d'aimer après Murambi?» (2008:186).

21 Entre la haine qui a guidé les massacres de masse et l'amour qui aurait pu freiner le génocide, V. Tadjo et $\mathrm{M}$. Ilboudo tentent d'insérer une réflexion sur le mal comme expression de l'inhumanité de l'Homme. En substrat de leurs réflexions, le génocide rwandais n'est pas un simple accident de l'Histoire, mais le résultat d'une négation de 
l'Homme sur la base d'un critérium socio-idéologique subjectivement entretenu et valorisé à des fins funestes.

Cette idéologie, construite à partir de stéréotypes et de préjugés très longtemps entretenus et enseignés dans des écoles, a fini par être servilement partagée par les uns et les autres, surtout par les hutu qui en ont fait un prétexte pour justifier le massacre de masse des Tutsi, aidés par la radio RTLM ou Radio Mille collines. Y. Mukagasana, une rescapée du génocide, rapporte une fiche signalétique du corps du Tutsi donnée à longueur de journée sur les ondes de cette radio de la mort, en ces termes :

Comment distingue-t-on le cancrelat du Hutu? Plusieurs moyens sont à votre disposition. Le cancrelat a les incisives écartées. Le cancrelat a le talon étroit. Le cancrelat a huit paires de côtes. La femme cancrelat a des vergetures sur les cuisses près des fesses. Le cancrelat a le nez fin. Le cancrelat a le cheveu moins crépu. Le crâne du cancrelat est long derrière, et son front est incliné. Le cancrelat est grand et il y a de la morgue dans son regard. Le cancrelat a une pomme d'Adam prononcée (Mukagasana 2000[1999] : 24-25).

Le fond idéologique de cette tragédie que V. Tadjo et M. Ilboudo voulaient " exorciser » réside dans la «fascination du Tutsi» (Semujanga 2008: 41) perçu par les Belges présents dans la région à la fin de la première guerre mondiale jusqu'à la fin des années cinquante, comme des "Blancs à la peau noire » avec « une intelligence supérieure " (2008: 45). Comment des critères morphologiques ont pu entrainer une violence aussi cruelle? Qu'est ce qui a pu justifier les dix commandements des Bahutus clairement dirigés contre les Tutsi?

L'un de ces commandements (le premier par exemple) problématise la condition de la femme tutsi, lorsqu'il indique fermement qu' « est traitre tout Muhutu qui épouse une Mututsikazi, qui fait d'une Mututsikazi sa concubine, qui fait d'une Mututsikazi sa secrétaire ou sa protégée ». Une posture idéologique qui traduit la dimension dramatique de l'histoire d'un peuple qui a construit son parcours autour de la haine et de l'altérité, de l'exclusion et de l'impensable, si bien que le « témoin » qui n'a " pas de langue " en voyant une telle inhumanité ", voit son "rôle [...] amputé", selon la formule de J. Kagabo (1999 : 75-78), de retour de son pays exsangue.

À travers les voix de leurs personnages et celles des victimes qu'elles ont rencontrées ici et là, $\mathrm{V}$. Tadjo et M. Ilboudo tentent de comprendre ce massacre de masse au moyen d'une écriture de l'accusation et de la révolte, qui problématise le rôle qu'aurait pu jouer l'écrivain dans la prévention ou l'empêchement du génocide contre les Tutsi du Rwanda. Le projet de V. Tadjo et d'E. Ilboudo rappelle la mise en garde de P. Nganang sur les dangers d'un oubli ou d'une négation éventuels d'un drame qui s'est bien produit aux yeux de tous, et qui ne devrait souffrir d'une quelconque forme de minimisation. Selon lui,

On ne peut plus écrire aujourd'hui en Afrique, comme si le génocide de 1994 au Rwanda n'avait jamais eu lieu [...] Tragédie la plus violente que l'Afrique ait connue ces derniers temps, il est aussi le symbole d'une idée qui désormais fait corps avec la terre africaine: l'extermination de masse perpétrée par des Africains sur des Africains (Nganang $2007: 24)$.

En s'inscrivant dans ce projet de mémoire tel que formulé par P. Nganang, les deux auteures restituent le génocide dans toute sa radicalité, s'efforçant d'instituer une réflexion contre l'oubli ou la négation, au moyen d'« une prise de parole difficile mais pas impossible» (Kagabo 2014: 5-8). La démarche consiste à porter la voix des survivants en se constituant témoins de leur histoire douloureuse, par les mots. C'est là 
tout l'enjeu d'un voyage au milieu des victimes meurtries par la douleur que leur plume tente de transcrire.

De fait, leur discours sur le génocide permet de comprendre que la haine est fondamentalement à la base de ces massacres de masse, que traduisent des désignations marquées par une altérité frappante comme «eux» et «nous». L'indicateur principal exprimant la construction de ce monde binaire et conflictuel divisé entre "eux» et "nous» (comme ce fut le cas durant le génocide rwandais), réside dans le discours idéologique qui définit celui qu'il faut exclure, voire tuer (le Tutsi, mais aussi le hutu-traitre) ou " eux », et celui qu'il faut épargner (le Hutu loyal) ou « nous ».

En partant au Rwanda, V. Tadjo avait une hypothèse: «ce qui c'était passé nous concernait tous. Ce n'était pas uniquement l'affaire d'un peuple perdu dans le cœur noir de l'Afrique ", (Tadjo 2000 : 13). Cette hypothèse, tout en stimulant le voyage au cœur de ce petit pays d'Afrique centrale, traduit un message que l'auteure ivoirienne veut faire passer: lorsque survient une tragédie dans un quelconque pays africain (comme le génocide rwandais), c'est toute l'Afrique qui devrait se sentir concernée.

Chez les deux auteures, le projet d'écrire par devoir de mémoire s'inscrit dans la tentative de comprendre et faire comprendre ce qui semble avoir été l'expression d'un nihilisme humain. Un nihilisme basé sur la haine de l'autre et la négation de son humanité, d'autant plus que les Tutsi étaient perçus comme des cafards à éradiquer de la surface du sol, des insectes puants et nuisibles qui ne méritent qu'une seule chose : la mort.

En effet, la propagande (bien planifiée par l'élite politique) qui a conduit au génocide s'est appuyée sur l'idéologie du peuple majoritaire que sont les Hutu dont l'arme est la machette et l'ami, la France, qui a pour obligation de débusquer et d'exterminer l'ennemi tutsi réduit à l'état de bête sauvage (cafard, vermine, serpent). Le bestiaire métaphorique auquel étaient associés les Tutsi traduit la radicalité du mal dont la phase ultime consiste à tuer l'ennemi tutsi qui ne mérite rien d'autre que la mort (dans l'imaginaire hutu), après qu'il a été assimilé à l'animal.

\section{Une écriture-femme ou la posture féministe dans le récit du génocide}

31 La problématique d'une écriture féminine dans l'approche des grands sujets de la société se présente moins comme une « théorie » que comme un questionnement global sur ce que c'est que le féminin ou l'affirmation du féminin. Selon S. de Beauvoir, « il est connu que la femme est bavarde et écrivassière » $(1976: 628)$, bien qu'elle ait tendance à saisir le monde à travers une vision singulière. Dans Speculum de l'autre femme (1974 : 176), L. Irigaray postule le caractère incontournable de la différence sexuée, s'efforçant de dénoncer la domination masculine, qui impose ses mots "sensés " « impuissants à traduire ce qui se pulse, clame, se défend, floué ».

Il se dégage, de plus en plus, une évolution de l'écriture-femme, de l'écriture au féminin théorisée par B. Slama, à l'instar de L. Irigaray, laquelle présente la posture subversive de la femme sortant des limites et territoires que lui impose la société, et qui met en scène des thèmes qui ont « une couleur particulière » (Slama 1981 : 51-71). 

quelques exceptions), la plume de certaines écrivaines africaines défendant la cause des femmes laisse cependant penser une présence féministe dans les écritures africaines modernes. Cette posture, qui remonte à des pionnières dont l'œuvre manifeste l'ambition féministe africaine naissante face aux traditions sociales et religieuses (nous pensons à M. Ba avec Une si longue lettre en 1979), semble influencer les auteures de la nouvelle génération. Leur parole, libérée, tente de faire écho aux cris des victimes de la violence armée.

Dans le creux du voyage jusqu'au bout du Rwanda, V. Tadjo et M. Ilboudo donnent la parole aux rescapés et aux victimes (dont un grand nombre de femmes), décrivent des scènes de tueries, de massacres et d'abus sexuels suivis de meurtres, le tout dans une atmosphère de haine. Leur récit du génocide va au-delà d'un simple compte rendu de guerre pour toucher essentiellement aux sujets se rapportant aux femmes.

$\mathrm{Du}$ fait de la banalisation de leur corps, certaines femmes ne croient plus en leur féminité marquée par la souillure et le déshonneur. Sous le poids de la douleur, l'une d'elles, dans le roman de M. Ilboudo, fait la grave révélation suivante : «Je ne suis plus une femme ». Le narrateur raconte l'histoire de cette femme comme suit :

Dans les premiers jours du génocide, elle avait été " 'retenue »' par un de ses voisins militaires, qui sous le prétexte de la protéger, l'avait séquestrée pendant trois semaines, abusant sans vergogne de son jeune corps. Lorsqu'elle a essayé de fuir cet enfer, il l'avait rattrapée et, pour la punir, l'avait livrée à un groupe de miliciens, une dizaine de brutes ivres et drogués, mais assez lucides pour se mettre à rang et la violer chacun à son tour. Comme distraction finale, l'un d'eux avait proposé d'introduire une bouteille pleine d'acide dans son sexe (Ilboudo 2002 : 74).

Le lecteur attentif est saisi par une attention portée aux femmes et à leurs conditions pendant le génocide, tant apparaissent des descriptions et des allusions touchant à leur situation dramatique. Derrière ces descriptions, se profile la violence comme point de suture entre l'occurrence de ces femmes et les actes dont elles sont victimes parce qu'elles sont, pour la plupart d'entre elles, tutsi, « un crime impardonnable » (Ilboudo $2002: 29$ ).

Le viol des femmes, à titre d'exemple, est très présent dans le récit. Arme de guerre pendant le génocide, les scènes qui le mettent en exergue ou en font allusion, parcourent ici et là le tissu narratif comme pour marquer le territoire du génocide et le forcer à s'y adapter. Parfois, le viol en lui-même ne semble pas suffire. Il peut être accompagné d'« un pic » qu'on enfonce dans le vagin (Tadjo 2000:20), du virus du «SIDA » que le bourreau transmet volontairement à ses victimes, dont quelques-unes sont « dépecées » après avoir été violées (Ilboudo 2002: 51), le corps souillé « jusqu'au fond de l'âme » (op cit : 70), la « féminité endolorie [...] bafouée » (op cit : 65).

Dans Avant de tuer les femmes, vous devez les violer (2014), S. Riccie montre le caractère monstrueux du viol durant le génocide rwandais pendant lequel les bourreaux avaient une obsession pour la «matrice » des femmes tutsi, qui, selon eux, n'était pas la même que celle des femmes hutu. Son analyse sur ce qu'elle appelle "les rapports sociaux du sexe " rejoint celle de V. Tadjo et de M. Ilboudo, à propos du viol comme arme de guerre. 

l'humanité, dès lors que le viol, qui est une forme genrée du génocide, est banalisé et perçu comme faisant partie des effets collatéraux de la guerre ; mais bien plus, comme une arme de guerre pour détruire psychologiquement l'autre et l'humilier.

les pages du récit du génocide, les femmes dont parlent V. Tadjo et M. Ilboudo s'inscrivent essentiellement dans la posture de victimes. Dans la majeure partie des cas, elles racontent elles-mêmes leur propre histoire, avec des mots d'une extrême crudité, lesquels occupent largement l'univers du texte. La narratrice dans le roman de M. Iboudo parle de femmes envahies "par la mémoire du viol», dont le corps (le sien notamment), du fait du viol et de la violence, «n'était que souillures et meurtrissures " (Ilboudo 2002 : 55). Elle décrit également des femmes traumatisées, dont «les crânes d'enfants en pleur " sont défoncés après que le bourreau les a arrachés « du dos de leurs mères en larmes » (op cit:51).

Dans cette posture du récit homodiégétique, la parole du témoin porte un message individuel et personnel à une collectivité, pour que la réception du récit du génocide ne souffre d'aucun doute. Les voix des personnages féminins (notamment les deux narratrices dans les deux romans) qui racontent le génocide s'inscrivent dans un élan d'attestation de la vérité, qui passe par la parole du témoin-victime, du témoin-direct (celui qui a assisté au massacre de masse, en a subi un dommage, un préjudice), comme c'est le cas d'Esther, survivante du génocide, qui raconte son parcours de rescapée avec la ferme conviction que de toutes les façons, le survivant est " condamné à vivre ", lui qui doit opter pour ce qu'elle appelle une « vie vivante » (Mujawayo $2005: 17$ ).

Même quand V. Tadjo évoque des femmes-bourreaux du génocide, c'est par pure incidence. En arrière-plan d'une telle posture double, on perçoit, en réalité, l'image de la femme-victime. Cette description du «Bloc 145 » d'une prison où sont entassées "deux cent cinquante-trois femmes », en témoigne :

Femmes meurtrières, femmes génocidaires, femmes contraintes à tuer, accusées d'avoir tué leurs époux, leurs enfants, des amis, des voisins, des inconnus. Femmes qui ont aidé des hommes à commettre des viols, qui ont chanté pour leur donner le courage de massacrer, qui ont trahi, qui ont pillé, qui ont décidé de se joindre à la cruauté. À coups de machettes, elles ont tué d'autres femmes, mutilé des enfants, achevé des hommes... Elles sont entrées dans les hôpitaux, les églises, les écoles pour participer au carnage. Elles ont pris l'argent des morts, les bijoux des femmes tombées, leurs habits (Tadjo $2000: 113)$.

En portant un regard double sur le rôle des femmes pendant le génocide, V. Tadjo et M. Ilboudo tentent le jeu de l'équilibre et de l'objectivité. Les deux auteures essaient de dépasser le dilemme de l'alternative entre ce qui semble être une évidence pour le commun des mortels (la femme-victime), et la réalité des faits (la femme victime et bourreau du génocide). La figure de la femme violée ou mutilée et celle de la femme meurtrière révèlent un double procès de solidarité et de déception.

Solidarité, parce qu'en tant que femmes, V. Tadjo et M. Ilboudo se sentent profondément concernées et touchées par la violence physique et psychologique dont sont victimes des femmes quand éclatent des conflits armés, la plupart du temps provoqués par des hommes. Déception, dès l'instant où des femmes ont encouragé (sous la contrainte des hommes) le viol et le meurtre d'autres femmes.

GLAD!, 06 | 2019 

témoignage et de l'engagement, les auteurs ayant participé à cette résidence d'écriture savaient qu'il fallait de la force dans les mots pour transcrire la violence dans toute sa radicalité. Ils savaient également que la douleur des morts qui ne peuvent plus parler et celle des rescapés sous le poids du choc, devaient être racontées avec un discours brut et indécent, en parfaite symbiose avec l'univers de la guerre. Ils avaient compris, en effet, qu'il fallait éviter le piège du réel inaudible du génocide qui est capable de résister à la crudité des mots. Même s'ils étaient pour la plupart des témoins « du dehors" (selon la formule de R. Fonkoua), ils avaient conscience de "hisser leur écriture à la hauteur de la souffrance » (Delas 2000 : 21). convoquant un univers guerrier avec ses inclinations choquantes, se devait de proposer ce que J. Semujanga appelle «l'espoir d'une vie possible après le drame grâce à la fiction " (2008: 218). Une telle démarche nécessitait de créer une passerelle entre le choc des mots pour traduire la violence et l'usage d'un lexique d'espoir, d'une écriture qui propose un monde meilleur.

50 En optant pour un langage violent et un lexique essentiellement dénotatif inscrits dans l'univers d'un réel occurrent, l'écriture du génocide postule une logique qui veut que la vérité de la guerre soit traduite par des mots canons, des mots qui épousent les stridences du récit véridique. Une écriture qui s'enracine, selon B. B. Diop (2001), « dans la réalité [...] qu'il faut aller chercher [...], le plus vite possible par les formes les plus désespérées ».

51 Les textes de V. Tadjo et de M. Ilboudo répondent aux critères de la parole libérée et frappent par la crudité des mots du génocide qui servent à transcrire la guerre entre le 
bourreau et sa victime, avec l'expérience de la mort comme tension du récit. Pendant que M. Ilboudo parle de «l'odeur de la mort qui planait déjà sur Kigali [la capitale du Rwanda] " (Ilboudo, 2002: 37), V. Tadjo montre que celle-ci, "devenue intenable», « flottait dans Kigali » (Tadjo $2000: 83$ ), et qu' « enragés », « les chiens se sont nourris de cadavres », pendant que « les oiseaux » s'acharnaient sur les « yeux des cadavres » (op cit : 37$)$.

La vision de V. Tadjo et d'E. Ilboudo s'inscrit dans le «principe de l'affabilité », dans la théorie du dicible défendue par Semprun, selon laquelle « on peut toujours tout dire [ou ce qui est humain peut être dit], car le langage contient tout» (Rinn 1998: 23). Même s'il se révèle limité face à un "réel inépuisable ", même si l'extrême violence du génocide réduit la capacité du texte à "dire le réel», le langage reste un outil important pour conjurer le drame de la mort.

Dans cette approche, M. Rinn propose que la question de l'indicible ou de l'ineffabilité (que suggèrent certains) soit perçue comme un art du langage (la technè) qui n'interdise pas la représentation (du génocide), mais qui le saisit en tant qu'expérience d'écriture à vivre. Cette expérience d'écriture du génocide fait appel à la vue et à l'ouïe pour comprendre et évaluer le drame des Tutsi dans sa dimension horrifique. Elle intègre de la puissance dans les mots pour représenter la violence, quand il s'agit de traduire notamment la souffrance de la femme «ligotée, violée, meurtrie " (Ilboudo 2002:65), qui a «honte » et se sent "sale, repoussante », à cause de son corps bafoué et souillé (Tadjo 2000 : 77).

54 Autour du binôme «vue » et « ouïe » (que traduisent les voyages de V. Tadjo et d'E. Ilboudo au Rwanda) qui centralise la compréhension du massacre des Tutsi, est née, en effet, une écriture qui envisage le génocide comme une expérience à transcrire sans limite des mots. Ce possible narratif alimente la représentation de l'horreur dans laquelle se noue et se dénoue une possibilité d'écriture. En fait, vu son caractère mortifère et horrifique, le génocide rwandais a nécessité une nouvelle approche de l'écriture dans la forme comme dans le fond, une manière de faire écho à P. Nganang sur la nécessité d'écrire désormais en ayant en mémoire le fait que le génocide rwandais ait vraiment existé.

V. Tadjo le reconnait en ces termes: "L'écriture de L'ombre d'Imana marque un changement dans ma façon de travailler. » (Tadjo 2002 : 196) La méthode privilégiée par la quasi-totalité des rédacteurs a consisté justement à faire des recherches documentaires, à lire des dépêches, à faire des interviews, avec comme résultat chez $\mathrm{V}$. Tadjo et d'E. Ilboudo, des textes marqués au coin par la violence, par des descriptions choquantes et des témoignages à couper le souffle. C'est essentiellement une écriture « du corps-féminin, par la femme elle-même » (Didier, $1981: 35)$ que G. Fremont appelle « un casse-texte » marqué par la « mise en mots d'elle-même » (1979: 315-330).

Mis à l'épreuve " en tant que lieu de formation d'un imaginaire " (Germanotta, 2016 : 73-100), le langage du génocide parcourt les lieux du massacre pour faire la mise «à jour ", la mise "à nu", la mise «en jeu» de la barbarie des hommes, au cœur de laquelle on découvre le corps féminin que $\mathrm{N}$. Etoke considère comme le «lieu de tension » du récit (2006: 43). Dans « l'horreur de la terre souillée » (Tadjo $2000: 21$ ), il ne fait aucune économie de mots pour décrire «des dizaines de milliers de corps desséchés » (Ilboudo 2002 : 57), « des cadavres, partout le long des routes» (Tadjo 2000 : 83), des têtes fracassées, des tas d'os, des amoncellements de cadavres... Il se veut poignant, direct, sans tabou, pour dire le génocide. Il s'efforce de répondre aux attentes 
du public (ou du lecteur), qui, selon J. Semujanga, «manifeste généralement beaucoup de méfiance envers le roman dont il souligne le caractère fictionnel incompatible avec la fonction de récit de témoignage » (2008: 144).

Tous les lieux du génocide (domiciles, églises, écoles, rivières etc.) sont passés au peigne fin pour en extraire des femmes violées et meurtries par la guerre, des corps mutilés et souillés, des enfants assassinés et même " la mort dénaturée par la cruauté " (Tadjo 2000 : 19). Ici, la narratrice évoque « une femme veuve» qui « vivait seule dans sa maison. Son mari était mort pendant le génocide et elle avait vu son voisin tuer son fils unique » (op cit : 47). Là, il est question d'une autre dont la "vie s'est arrêtée un jour de juin 1994 " quand, "fugitive, donc fautive", elle est «tombée » sur ses "tortionnaires", notamment " un groupe de paysans qui revenaient sans doute d'une opération de nettoyage d'une colline voisine » (Ilboudo $2002: 45$ ).

Pour marquer d'une tâche forte l'horreur du massacre, certains faits sont transcrits avec le souci de faire sortir le lecteur de son mutisme et de son incrédulité, comme quand E. Mukakayumba parle des «mutilations des corps visant spécialement des organes liés à la reproduction ", notamment de l'« ouverture des corps des victimes [les femmes tutsi] du sexe au cou " (1995: 145-154). Selon M. A. Germanotta, une telle démarche visant à refuser de rendre l'horreur «scandaleusement tolérable " (2016: 73-100), est une façon de " restituer un visage à une souffrance rendue anonyme par le crime de masse ». Le récit du génocide inaugure ainsi un programme d'écriture capable de faire voir, de faire entendre et de faire sentir l'horreur du massacre grâce à un langage cru. Cet extrait que livre la narratrice de M. Ilboudo en témoigne :

Atteint du SIDA, un jeune chirurgien s'était laissé convaincre par un marabout que sa guérison passait par un remède à base de cœur pur encore palpitant. Il avait donc enlevé une fillette de treize ans, et dans sa cuisine, l'avait bâillonnée et attachée. Puis sans anesthésie, il lui avait ouvert le thorax avec des gestes précis de professionnalisme et avait arraché le cœur battant » (Ilboudo, 2002:68).

59 Ce passage, au-delà de la forte émotion qu'il suscite, révèle le caractère inhumain des génocides qui sont le lieu de toutes les dérives, de l'impensable, de ce que la narratrice de Murekatété appelle le "spectacle indigne de notre humanité offensée " (Ilboudo 2000 : 65). Le langage, par le choc des mots et par la violence de la description, est une réponse à la violence de la guerre qui veut, selon C. Coquio, que lui soit proposée « une construction dramatique ou tragique, une scénographie de la mort et du mal, pour que ce mal soit perçu » (Coquio $2004: 103$ ).

\section{Du chaos du génocide au discours de l'espoir}

En général, le génocide est porté par une construction acerbe et tranchée de l'écriture qui essaime un discours de la mort. Certains critiques croient ainsi assumer la responsabilité du discours militant qui exprime au mieux la douleur des victimes et le deuil des morts. Cependant, dans le tumulte de la folie meurtrière du Rwanda, est née, à côté de ce qu'A. Waberi désigne comme étant une Moisson de crânes (2000), une écriture de l'espoir qui est une contre-photographie de «l'Afrique-cauchemar ».

61 On note cette écriture de l'espoir dans « des chansons de Dieu » que fredonnent, pour se donner du courage, plusieurs femmes victimes du génocide ou dans des appels à la 
retenue et à la tolérance, comme celui du devin qui conjure les morts: «Hommes, femmes, prenez garde au désir de vengeance et au cycle perpétuel de la violence et des représailles » (Tadjo $2000: 112,59$ ).

Le but d'une telle stratégie consiste à inscrire l'évènement raconté dans l'espoir postgénocide, dès lors que pour V. Tadjo, comme l'indique la quatrième de couverture de son texte narratif, "la question du pardon est essentielle à la reconstruction». L'écriture, en participant au "travail de guérison d'un mal causé par l'horreur ", devient «thérapeutique » (Semujanga 2008 : 182) dans le processus de réparation des effets du génocide.

M. Ilboudo, par exemple, convoque le mythe d'Abel et Caïn pour construire son texte, dans une perspective de comparaison entre la haine qui pousse le frère ainé (Caïn) à assassiner son frère cadet (Abel), et celle à l'origine de la violence entre Hutu et Tutsi. Sous l'ombre d'Imana (Dieu en kinyarwanda), V. Tadjo retrace la fraternité brisée et rappelle que le sort qui s'est abattu sur le Rwanda ne peut être conjuré que par une inflexion fraternelle et spirituelle au centre de laquelle se trouve Dieu. En évoquant un récit de la Bible qui rappelle une situation de mort, « un crime resté impuni » (Ilboudo 2002 : 27), le récit du génocide rwandais problématise la question de la foi religieuse qui semble se perdre dans des violences fratricides comme celle qui ont opposé les Hutu au Tutsi, et ouvre la réflexion sur le sens de la fraternité.

Les écrits de V. Tadjo et de M. Ilboudo frappent (justement), à certains endroits, par un discours post-génocide optimiste, si on s'en tient au contre-discours sur la haine et le pessimisme, et sur ceux que l'imaginaire populaire désigne comme kabuhariwe (« ceux dont on n'attend plus un signe de reconnaissance d'humanité »). Cet espoir d'une vie nouvelle après la mort va même jusqu'à convoquer un univers surréel et onirique, dans lequel les morts semblent vaincre la mort, comme rapporté par la narratrice de L'ombre..., en ces termes :

Les morts renaitront dans chaque parcelle de vie aussi petite qu'elle soit, dans chaque parole, chaque regard, chaque geste aussi simple qu'il soit. Ils renaitront dans la poussière, dans l'eau qui danse, dans les enfants qui rient et jouent en tapant des mains, dans chaque grain caché sous la terre noire. Et les esprits partiront là où ils le désirent, non plus comme les âmes en peine mais comme des rayons-éclairs (Tadjo $2000: 57$ ).

65 Dans cet extrait, V. Tadjo transporte le lecteur dans un monde irréel qui trahit une vision idéologique sur le sens de la vie après la mort. Bien qu'elle soit « naturelle », la mort « est l'autre face de la vie » selon l'auteure. Pour cette raison, poursuit-elle, « il ne faut pas en avoir peur » car «de toute façon, la mort n'est pas plus forte que la vie », celle-ci finissant par « reprendre le dessus » (op cit : 135).

Face à l'horreur, le texte du génocide s'efforce de se tourner vers un discours de l'espoir dans lequel on découvre l'autre face du discours génocidaire : l'optimisme. "Penser » le génocide par des mots-espoirs, des mots-optimistes, tel est l'enjeu qui soutient une écriture du refus face au crime de masse. Une écriture qui s'attache à rechercher le sens de la vie après le génocide, par l'entremise de la parole du témoin soucieux de lutter à la fois contre l'oubli et le désespoir.

67 Si les deux auteures convoquent la justice dans le procès post-génocide, c'est justement pour prendre la défense des victimes, notamment les femmes comme elles. Elles veulent que justice soit rendue avant toute forme de réconciliation. V. Tadjo dans 
L'ombre d'Imana, par exemple, milite pour le tribunal coutumier, "le Gacaca»; une sorte de recherche "dans le passé des solutions pour le présent ", selon la définition que donne la narratrice, « car avec la justice officielle, il faudrait plus de cent ans pour juger tous les prisonniers », conclut-elle (V. Tadjo $2000: 108$ ).

Le combat contre ce que C. Coquio appelle «la déréalisation » $(2004: 180)$ est soutenu par un imaginaire positif de ce que peut redevenir le Rwanda : un pays de paix dans lequel vivent des communautés fraternelles. En dehors d'un discours à charge, marqué notamment par ce qu'elle désigne comme " une scénographie de la mort et du mal, pour que ce mal soit perçu » (2004:103), on assiste à un travail de deuil sous les traits d'un nouveau départ que l'écriture tente d'instituer. C'est pourquoi le discours de la mort s'efface quelquefois pour faire place au discours de la réparation dans lequel même les morts sont invités à jouer un rôle crucial, dès l'instant où «le bien n'a pas disparu, n'a pas été enterré dans les fosses communes » (Tadjo 2000 : 131).

Le vivre-ensemble est encouragé et le discours semble installer dans la mémoire des Africains, des formes de résiliences face à d'éventuelles crises sociales et politiques. Une manière d'inscrire le «plus jamais ça » dans l'imaginaire post-génocide (rwandais) que résume ce questionnement de M. Ilboudo :

Face aux guerres et aux conflits qui se répètent sur le continent africain, face particulièrement à ce terrible génocide du Rwanda, pouvons-nous nous limiter au seul acte de création? N'avons-nous pas une obligation morale à nous impliquer plus, à nous impliquer autrement? Pourquoi sommes-nous devenus aphones face à nos propres tragédies? (Ilboudo 2009 : 235-241)

Comme un point de départ, le souci de faire prendre conscience s'insère dans le récit du génocide. Il s'agit à la fois d'opter pour ce que K. Lamko (2000) appelle « la parole du fantôme ", et de jouer sur la corde sensible et indispensable du discours du renouveau et de l'espoir. En adoptant le profil de messagers de l'espoir, V. Tadjo et M. Ilboudo donnent une autre lecture du génocide rwandais.

71 Leurs textes narratifs s'inscrivent dans une démarche thérapeutique, dans la perspective de la guérison post-génocide par l'écriture, sans laquelle les morts, dont " certains étaient si furieux qu'ils refusaient de repartir quand venait le moment de quitter la terre", viendront hanter les vivants (Tadjo 2000: 53). Les paroles de conclusion de L'ombre d'Imana, qu'on retrouve dans les prières de sœur Agathe, confirment ce discours post-génocide de l'espoir qui résume les réflexions des auteures au lendemain du massacre de masse :

Déposez les armes. Laissez les plaies guérir et se cicatriser [...] Lorsque le temps aura déposé du baume sur nos peines et que les générations pourront enfin relever la tête, l'histoire de tous ceux qui ont su garder leur humanité sera dite et redite, racontée en susurrant les mots, chantée à pleine voix, dansée, célébrée [...] Nous sortirons de cette longue et terrifiante éclipse (Tadjo $2000:$ 130-131).

Le regard du témoin indirect s'efface derrière celui de l'auteure non-victime qui cherche à faire oublier la douleur des amoncellements de cadavres. C'est en fait « un engagement d'une forme nouvelle ", selon I. Gom (2013), marqué par une implication dans la recherche de solution à une tragédie que seuls les mots qui servent à la transcrire sont capables de la panser. 


\section{Conclusion}

Beaucoup ont craint l'étouffement des textes narratifs sur le génocide rwandais par une surenchère de l'écriture tournée vers la pratique de l'art pour l'art. Une telle stratégie aurait voilé l'horreur de l'évènement qui, comme la shoah, «remet en question la notion même d'homme " (Wardi 1986: 7). M. Ilboudo et V. Tadjo ont évité ce piège de l'anéantissement de la vérité dicible. Leurs textes frappent par ce que M. S. Jensen (2007) appelle "l'intimisme" marqué par une description poignante et intime du génocide où «le rescapé transforme le mutisme forcé qu'impose l'évènement en langage » (E. Brezault $2003: 7)$.

Dicible, le génocide rwandais est perçu par les deux auteures comme un objet social qui mérite une parole du témoin rendue palpable et compréhensible par la force des mots. Raconté avec une ferme envie de choquer en vue de pousser à l'action, le génocide des Tutsi invite à une relecture d'un événement qui induit une stratégie de survie des rescapés, des victimes et même des morts, que le langage tente de rendre possible.

Bien qu'étant des témoins indirects du génocide, $\mathrm{V}$. Tadjo et $\mathrm{M}$. Ilboudo ont écrit des textes dans lesquels la parole de femmes sous le choc fait écho à celle des victimes et des rescapés incapables de se soustraire tout seuls de la terrible douleur morale d'avoir perdu un conjoint, un parent, un enfant, un ami, un voisin.

Cette réflexion a permis de mettre en relief le langage du génocide selon deux auteures africaines francophones. Leur lecture de l'événement est à la fois un cri de cœur face à la haine des hommes, et un appel à l'amour pour conjurer la mort et la pensée revancharde. Comme toutes les œuvres fictionnelles sur le génocide du Rwanda, qui institutionnalisent «les lieux d'intersection où habite l'objet testimonial, hors d'un genre exclusif» (Karegeye 2009: 21-32), leurs textes injectent aux mots un contenu social, dans lequel s'imbriquent engagement féministe, témoignage pathétique et discours sur l'avenir.

\section{BIBLIOGRAPHIE}

BA, Mariama. 1979. Une si longue lettre, Abidjan : NEA.

BONNET, Véronique et SEVRAIN, Emilie. 2008. «Témoignages de rescapées rwandaises : modalités et intentions ", Ipotesi. Revista de estudos litérarios, vol. 18, pp. 105-113.

BREZAULT, Eloïse. 2003. « Raconter l'irracontable : Le génocide rwandais, un engagement personnel entre fiction et écriture journalistique » Éthiopiques. $n^{\circ} 71$, pp. 1-25.

CAZENEUVE, Odile. 1996. Femmes rebelles. Naissance d'un nouveau roman africain au féminin, Paris : L'Harmattan.

CIXOUS, Hélène. 1975. Le Rire de la Méduse et autres ironies (préface de Frédéric Regard), Paris : Galilée.

COQUIO, Catherine. 2004. Rwanda : Le réel et les récits, Paris : Editions Belin. 
DEEPIKA, Bahri. 2010. « Le féminisme dans/et le postcolonialisme » in Christine Verschuur, Genre, postcolonialisme et diversité de mouvements de femmes, Cahiers Genre et Développement, $\mathrm{n}^{\circ} 7$, Genève, Paris : EFI/AFED, L'Harmattan, pp. 27-54.

DELAS, Daniel. 2000. Écrits du génocide rwandais, $\mathrm{n}^{\circ} 142$, octobre-décembre, Paris : Notre Librairie.

ETOKE, Nathalie. 2006. « Ecriture du corps féminin dans la littérature de l'Afrique francophone : taxinomie, enjeux et défis », CODESRIA Bulletin, $n^{\circ}$ 3, 4, pp. 45-47.

FAULKNER, Morgan. 2012. "L'auteur face au génocide : la mise en scène de la subjectivité dans L'ombre d'Imana : voyages jusqu'au bout du Rwanda de Véronique Tadjo », Études romanes de Brno, 33, 1 : pp. 253-255.

FUSASCHI, Michela, 2013. «Le silence se fait parole. Ethnographie, genre et superstes dans le post génocide rwandais», Archivio Antropologico del Mediterraneo, XVI, n. 15 (2), pp. 29-40.

FREMONT, Gabrielle. 1979. « casse-texte », Études littéraires, vol. 12, n³ : pp 315-330.

GALLIMORE, Béatrice Rangira. 2001. «Écriture féministe ? Écriture féminine ? : Les écrivaines francophones de l'Afrique subsaharienne face au regard du lecteur/critique ", Études françaises : 37 (2), 79-98.

GALLIMORE, Béatrice Rangira. 1997. L'œuvre de Calixte Beyala, Paris : L'Harmattan.

GEFEN, Alexandre et BOUJU Emmanuel, 2014. L'émotion, puissance de la littérature ? Pessac : PU de Bordeaux, Raison publique/1 ( $\left.{ }^{\circ} 18\right)$, pp. 221-229.

GERMANOTTA, Maria Angela. 2016. «L'écriture de l'inaudible. Les narrations littéraires du génocide au Rwanda », Interfrancophonies $n^{\circ} 7$, Nouvelles formes de l'engagement dans les littératures francophones : pp.73-100.

GOM, Issa. 2013. « Nouveaux paradigmes narratifs dans les récits du génocide rwandais du fest'africa » Éthiopiques $\mathrm{n} \mathrm{n}^{\circ} 90$. Littérature, philosophie et art, Penser et représenter l'ethnie, la région, la nation [En ligne], consulté le 31 Juillet 2018, URL : http://ethiopiques.refer.sn/ spip.php?article1859.

ILBOUDO, Monique. 2000. Murekatete, Bamako : Le figuier.

IRIGARAY, Luce. 1974. Speculum de l'autre femme, Paris : Ed de Minuit.

JENSEN, Merete Stistrup. 2000. « La notion de nature dans les théories de l'“écriture féminine”, Clio. Femmes, Genre, Histoire [En ligne], consulté le 10 octobre 2018, URL : http:// journals.openedition.org/clio/218 ; DOI : 10.4000/clio.218.

KAGABO, José. 1999. "Pas de langue pour l'hébétude", Le travail de mémoire. Une nécessité dans un siècle de violence, Coquio, Cath. (dir.), Paris : Autrement, pp. 75-78.

KAGABO, José. 2014. "Le génocide des Tutsi, comment penser une 'barbarie' en apparence aveugle”, Les temps modernes, n680-681, Paris, Gallimard, pp. 5-18.

KAREGEYE, Jean-Pierre. 2014, "Les femmes témoins : la prise de parole”, Les temps modernes, $\mathrm{n}^{\circ} 680-681$, Paris : Gallimard.

KAREGEYE, Jean-Pierre. 2009. "Rwanda : Littérature post-génocide, écritures itinérantes : témoignage ou engagement?». Protée, vol. 37, n² : pp. 21-32.

KOULSY, Lamko. 2000. La parole des fantômes (entretien), propos recueillis par Sylvain Marcelli, "L'Interdit", [En ligne], consulté le 12 Septembre 2018, URL : http: //insite.fr/interdit/2000nov/ rwanda6.htm. 
LECLERC, Annie. 1974. Parole de femme, Paris : Grasset.

MONGO-MBOUSSA, Boniface. 2002. Entretien avec Véronique Tadjo, Désir d'Afrique, Paris : Gallimard.

MUJAWAYO, Esther et BELHADDAD, Souâd. 2005. SurVivantes : Rwanda - Histoire d'un génocide, Paris : Ed. de l'Aube.

MUKAGASANA, Yolande. [1999] 2000, N'aie pas peur de savoir, Paris : J'ai lu.

MUKAKAYUMBA, Edith. 1995, "La violence faite aux femmes en contexte de conflit armé généralisé au Rwanda”, Recherches féministes, vol. 8, n. 1, pp. 145-154.

MUKAYIRANGA, Speciosa., 2003, “Sentiments de rescapés”, in C. Coquio, a cura, L'histoire trouée. Négation et témoignage, Nantes : Libraire l'Atalante, pp. 776-788.

NGANANG, Patrice. 2007. Manifeste d'une nouvelle littérature africaine : pour une écriture préemptive, Paris : Homnisphères.

OUEDRAOGO, Angèle Bassolé. 1998. "Et les Africaines prirent la plume. Histoire d'une conquête !", Mots pluriels, no 8.

RIGOLT, Bruno. 1974. “Écriture féminine et revendication identitaire”, Étude d'un extrait de Paroles de femme d'Annie Leclerc, [En ligne], consulté le 13 Octobre 2018, URL : http:// brunorigolt.blog.lemonde.fr/2016/03/12/annie-leclerc-parole-de-femme-texte-explique-lectureanalytique-eaf/\#ixzz5FrN68Wqx.

RINN, Michael. 1998. Les récits du génocide. Sémiotique de l'indicible. Lausanne : Delachaux et Niestlé. ROCHEFORT, Florence, 2018, Histoire mondiale des feminisms, Paris : Presses Universitaires de France, Coll. Que sais-je?.

SLAMA, Béatrice. 1981. De la "littérature féminine" à "l'écrire-femme" : différence et institution, Littérature $\mathrm{n}^{\circ} 44$, pp. 51-71.

SARTRE, Jean Paul. 2002. Qu'est-ce que la littérature? Paris : Gallimard.

SEMUJANGA, Josias. 2008. Le génocide, sujet de fiction? Analyse des récits du massacre des Tutsi dans la littérature africaine, Montréal, Edition Nota bene

TADJO Véronique. 2000. L'ombre d'Imana, Voyage jusqu'au bout du Rwanda, Paris : Actes sud.

TISSERON, Serge. 2011. Communications $\mathrm{n}^{\circ}$ 88, Paris : Seuil, pp. 83-91.

WABERI, Abdourahame. 2000. Moisson de crânes, Paris : Le Serpent à Plumes.

WARDI, Charlotte. 1986. Le génocide dans la fiction romanesque : Histoire et représentation, Paris : PUE. WITTIG, Monique. 1973. Le corps lesbien, Paris : Edition de Minuit.

\section{RÉSUMÉS}

On assiste de plus en plus à l'émergence d'un féminisme africain dans le roman. Des auteures africaines y ont trouvé un espace fertile pour exprimer leurs sentiments face à des sujets comme les génocides. Celui du Rwanda en 1994 permet de comprendre les paroles qui divisent et les dangers qui guettent notre humanité. Cette étude analyse le discours féministe sur cette tragédie. Elle montre comment Véronique Tadjo dans L'ombre d'Imana. Voyage jusqu'au bout du Rwanda et Monique Ilboudo dans Murekatété, dans un langage de révolte, avec des mots crus, présentent un féminisme révolutionnaire. 
There is growing evidence of the emergence of African feminism in novels. African writers have found a fertile space to express their feelings about topics like genocides. That of Rwanda in 1994 allows us to understand the words that divide and the dangers that lie in wait for our humanity. This study analyzes the feminist discourse on this tragedy. It shows how Véronique Tadjo in The shadow of Imana. Journey to the end of Rwanda and Monique Ilboudo in Murekatété, in a language of revolt, with raw words, present a revolutionary feminism.

\section{INDEX}

Thèmes : Recherches

Mots-clés : discours féministe, féminisme africain, féminisme révolutionnaire, génocide

Keywords : feminist discourse, African feminism, revolutionary feminism, genocide

\section{AUTEUR}

\section{DIDIER BROU ANOH}

Didier Brou Anoh est docteur en littérature francophone et exerce comme enseignant-chercheur à l'Université Félix-Houphouët Boigny (Côte d'Ivoire). Ses travaux de recherches portent notamment sur les nouvelles écritures et le renouvellement de l'écriture romanesque d'Afrique subsaharienne francophone, les questions du genre et de la mobilité. 